\title{
PROPUESTA UNIVERSITARIA DE EDUCACIÓN EN COLOMBIA: "PROFESIONAL DE LA VIDA COMUNITARIA Y LA PAZ"
}

\author{
Lyla Piedad Velosa Amature1, César Maurício Junca Rodríguez1 y Jonh Harold Estrada \\ Montoya 1
}

1Universidad Nacional de Colombia, Colombia. Ipvelosy@gmail.com; cesarjunca@autistici.org; jhestradam@gmail.com

\begin{abstract}
Resumen. Introducción: La educación comunitaria tradicional ha desconocido a los sectores populares por considerarlos ignorantes y los responsabiliza y culpabiliza por su mala salud. Sus modos de organización que les permiten resolver los problemas diarios con saberes y prácticas propias, han sido poco exploradas. Objetivos: Proponer un proyecto educativo para la vida y la paz desde una praxis transformadora. Métodos: Investigación cualitativa crítico-hermenéutica, fundamentada en la Investigación Acción Participativa y en la Educación Popular. Se sistematiza un proyecto de extensión solidaria anual realizado por casi 20 años por profesores y estudiantes de una Universidad colombiana con comunidades urbanas, indígenas y rurales. Se realizaron 12 historias de vida y se acompañó a 25 estudiantes en sus trabajos de grado en temas relacionados con dicha propuesta. Resultados: Se presentan las reflexiones de las historias de vida y del acompañamiento de la Universidad a comunidades marginalizadas que cristalizan en el Programa del Profesional de la Vida Comunitaria y la Paz. Conclusiones: La salud pública debe acercarse a los sectores populares desde un horizonte de sentido político particular y reconocer el saber popular como una forma de construcción de mundo con la que podrá ampliar su perspectiva de aproximación y de trabajo.
\end{abstract}

Palabras clave: Investigación Cualitativa; Agentes Comunitarios de Salud; Participación Social; Efectos Colectivos de las Desigualdades en Salud; Educación en Salud.

\section{UNIVERSITY EDUCATION PROPOSAL IN COLOMBIA: "PROFESSIONAL OF COMMUNITY LIFE AND PEACE"}

\begin{abstract}
Introduction: Traditional community education has ignored popular sectors because they consider them ignorant and blames them for their poor health. Their modes of social organization that allow them to solve daily problems with their own knowledge and practices have been little explored. Objectives: To propose an educational project for life and peace from a transformative praxis. Methods: Hermeneutic critical qualitative research, based on Participatory Action Research and Popular Education. An annual solidarity extension project carried out for almost 20 years by professors and students of a Colombian University with native, urban and rural communities is systematized. 12 life stories were made and 25 students were accompanied in their degree work on topics related to the proposal. Results: The reflections of life stories and the accompaniment of the University are presented to marginalized communities that crystallize in the Community Life and Peace Professional Program. Conclusions: Public health must approach popular sectors from a particular political sense horizon and recognize popular knowledge as a way of building the world with which it is necessary to broaden their approach and work perspective.
\end{abstract}

Keywords: Qualitative Research; Community Participation; Socioeconomic Factors; Health Education; Curriculum.

\section{INTRODUCCIÓN}

Los programas educativos en el área de la salud, en relación con la extensión universitaria, tanto de carácter preventivo de la enfermedad como de promoción de la salud se han realizado tradicionalmente desde la lógica y los criterios del paradigma de la medicina 
científica que no brinda una mirada integral en la comprensión de la salud. Dichos programas no han tenido suficientemente en cuenta la cultura, los intereses, lógicas y condiciones económicas de los sujetos a quienes se pretende hacer cambiar de hábitos, costumbres o supuestamente sacar de la ignorancia en la que se hallan y que los ha llevado a vivir en las condiciones de vida en las que se encuentran, desconociendo que son las condiciones estructurales económicas, sociales, históricas, culturales y políticas las que han hecho que el sector popular viva como vive, sea lo que es y exista en las condiciones que existe y no de otra manera, como suponen los profesionales que abordan las comunidades con el ánimo y la buena intención de brindar mejor salud, esperando que con campañas educativas, brigadas de salud y programas de extensión realizadas de manera intermitente en las comunidades, según los tiempos académicos, se resuelvan los malos hábitos en salud y se adquieran las costumbres necesarias para prevenir la enfermedad, sin que se incida en los verdaderos problemas que producen la enfermedad (Quevedo, 1992).

Es decir, que si el concepto de salud se reduce a un estado, a no estar enfermo, a no tener signos y síntomas ya clasificados y a evitar los factores causales individuales o del medio ambiente, se desconoce que el mundo de lo social y cultural brinda un espacio para la comprensión de la vida como un proceso histórico y político que abre infinitas puertas de explicaciones y soluciones enmarcadas en la promoción de la vida con calidad y en dignidad. El estilo de vida impuesto y extendido por el modo de producción capitalista determina los criterios y las condiciones para definir lo que es la salud y la enfermedad. Entonces, tendríamos salud capitalista, enfermedad capitalista y salud pública capitalista. Que es una forma de vida pero que aparece como si fuera la única forma de vida. En el modo de vida del capitalismo hay otras formas de vivir que, aunque no consideradas, son el diario vivir de la mayoría de los pueblos en la tierra. En Latinoamérica la mayoría vive en condiciones de pobreza y con limitaciones materiales inmensas. Estas condiciones en que viven las comunidades, son generadas por prácticas sociales, culturales, históricas y políticas de exclusión que están generalizadas en el Continente como modos de relación social dominante.

Las comunidades, tienen modos de organización social que les permiten resolver los problemas diarios con un saber y prácticas propias. Esas condiciones son las que mediante procesos de acompañamiento e intervención, hemos querido comprender y ayudar a transformar si fuese necesario. Ese saber es el que reconocemos en esta investigación como el saber de los sectores populares, de las clases populares de Latinoamérica. 
En el año de 1997 la Facultad de Odontología de la Universidad Nacional de Colombia implementó un nuevo plan de estudios con el que se logró un avance en la formación de los estudiantes por el criterio integrador, buscando superar la desarticulación entre la Facultad y otras carreras de la Universidad, entre la teoría y la práctica, entre lo individual y lo colectivo, entre la docencia y la investigación; igualmente, permitió enfatizar por primera vez de manera diferente en el componente comunitario a través de una propuesta denominada "Línea de Profundización Salud Familiar y Comunitaria", brindada a los estudiantes del último año de la carrera, que pueden elegirla de manera optativa entre 6 propuestas diferentes, disponibles en la Facultad. Para esta investigación, los estudiantes que durante estos casi 20 años han cursado la línea de profundización, se convierten en actores imprescindibles por sus aportes en investigación e interacción con las comunidades urbanas y rurales, campesinas e indígenas con que hemos trabajado.

Gracias al acumulado educativo e investigativo de los investigadores principales de este proyecto, que tiene un fuerte énfasis en extensión solidaria, es que se ha podido llegar al nivel de desarrollo que se tiene en el terreno del trabajo con comunidades rurales y urbanas empobrecidas y con grupos indígenas, acumulado que nos permite haber construido una propuesta de comprensión y de interacción con dichas comunidades que transforma el asistencialismo y contribuye, desde el sector educativo, a la superación de la pobreza, la injusticia social y los enormes niveles de desigualdad que han hecho tan doloroso el proyecto de vida en dichos sectores. La contribución principal desde la Universidad Pública, se centra en la construcción de un proyecto educativo comunitario de promoción de la vida y de la paz, con los sectores populares, urbanos y marginales y las comunidades indígenas, a partir de sus propios y principales intereses, de sus prácticas y escenarios cotidianos y de sus dinámicas de autogestión y desarrollo comunitario, con el ánimo de contribuir a la salud a través de la elevación de la calidad de vida de las comunidades y la formación humanística de los futuros profesionales, superando así el reducido espacio que en salud oral se ha trabajado tradicionalmente y que limita de marcada manera el accionar tanto teórico como práctico a la cavidad oral cuando no únicamente a los dientes.

Ha llegado el momento de abordar otras metodologías como las que brindan los espacios interdisciplinarios y que recogen los trabajos de investigación y extensión solidaria, ya realizados y que están más acordes con las circunstancias actuales colombianas y 
latinoamericanas (Mejía, 1999). Es, en el terreno de la educación, desde las opciones transdisciplinares, contextualizando críticamente las teorías, con prácticas comunitarias y a la luz de lo que conocemos como los paradigmas emergentes (Bautista, 2014; Carr, 1988), que nos disponemos a construir una praxis desde lo social que permita superar las limitaciones del biologismo, del funcionalismo, del academicismo y del conductismo para lograr una acción transformadora en relación con la naturaleza y con los demás hombres superando la díada salud-enfermedad y enriqueciendo con categorías mucho más amplias como: calidad de vida, cultura, vida cotidiana, género, lenguaje, trabajo, ingreso y salud colectiva y comunitaria que contribuyen a la promoción de la vida en dignidad y con ésta a la construcción de condiciones sociales más justas, equitativas y legítimas que redundarán en la consolidación de una vida saludable para los colectivos y comunidades colombianas (Mariño, 1992; Mariño, 1994).

El saber en la construcción de la vida colectiva tiene otras maneras de hacerse, parte de lo cotidiano, la práctica, lo compartido, lo contradictorio y lo imprevisto. Ese modo de creación del conocimiento tiene una historia, su trayectoria (Mejía, 1999). Es un saber que parte de la acción humana concreta sobre la cotidianidad, que cambia según las condiciones sociales, culturales, económicas y políticas en un momento y lugar específicos y que se hace desde el interés y la situación de los sectores populares (Freire, 1975; Freire, 1997). La propuesta de salud en paz se acerca a una perspectiva de conocimiento creada en el sur global como un aporte de Latinoamérica a las epistemologías del Sur (De Soussa, 2014; Bautista, 2014). Ante las condiciones de pobreza y exclusión en que se encuentran los sectores populares e indígenas que conllevan a altas tasas de mortalidad, niveles elevados de enfermedad general y oral y por ende una baja calidad de vida, cabe la pregunta: ¿Cómo reorientar los programas educativos universitarios de trabajo comunitario desde el área de la salud, para incidir positivamente en la promoción de la vida y la paz en los sectores indígenas, populares-urbanos-marginales e implementarlo en una sede de la Universidad Nacional de Colombia?

\section{OBJETIVOS}

Contribuir con una propuesta educativa desde la educación superior -partiendo del contexto económico, político y social colombiano- a la promoción de la vida comunitaria y de la paz con los sectores indígenas, populares-urbanos-marginales, recogiendo la experiencia de la praxis transformadora que durante los pasados 20 años ha venido construyéndose desde la 
Facultad de Odontología por profesores y estudiantes con los sectores populares de diferentes regiones del país.

\section{METODOLOGÍA}

Esta investigación es de corte cualitativo con un carácter crítico, hermenéutico, es decir, interpretativo y con un sentido educativo prospectivo, inscrita epistemológicamente en las corrientes de la Investigación-Acción-Participativa (IAP) y la Educación Popular (EP). Bajo la denominación de enfoque cualitativo de investigación social educativa se entienden todas aquellas metodologías orientadas a describir e interpretar críticamente determinados contextos y situaciones de la realidad social, buscando la comprensión de la lógica de sus relaciones, así como las interpretaciones dadas por sus protagonistas, con el ánimo de lograr transformaciones que permitan elevar las condiciones de vida de los individuos en los que se centra la investigación (Arnal, 1992; Bonilla, 1993; Bouché, 1994; Carr, 1988; Delgado, 1994; Goetz, 1988; Mariño, 1994; Mejía, 1999; Taylor \& Bogdan, 1986).

Los anteriores rasgos se aproximan a lo que en la clasificación hecha por Habermas para la investigación en las ciencias sociales son las metodologías histórico-hermenéuticas y críticosociales (Habermas, 1987). De manera muy general podemos afirmar que, la academia tiene los conocimientos disciplinares, es letrada e institucional; los saberes de los sectores populares son fundamentalmente orales, tradicionales y locales. Para que estas dos grandes realidades, academia y sectores populares, encuentren la capacidad de dialogar, necesariamente debe aflorar el infinito mundo de la cultura. Como académicos, para cumplir con los principios de la I.A.P, hemos utilizado herramientas de investigación como la etnografía multisituada, la autoetnografía, la observación participante, las entrevistas estructuradas, las historias de vida, entre otras, para desentrañar, reconocer e interpretar las lógicas que subyacen a las prácticas sociales para entablar los tan conocidos diálogos de saberes e interculturales (Fals Borda, 1999; Goetz, 1988; Molano, 1998). En el componente de la Acción y la Participación, ha sido indispensable que los futuros profesionales, tengan o adquieran una voluntad, un gusto por su quehacer comunitario, que es indispensable para lograr la puesta en marcha del trabajo a través de la educación popular realizada con las comunidades participantes. La Educación Popular es: política, intercultural; promotora de la convivencia; defensora de la paz; contextual; dialógica y crítica. Nadie sabe todo, nadie ignora todo, no existe la tábula rasa en los actores de los procesos educativos (Freire, 1975; Freire, 1997). La I.A.P es una forma de conocimiento que implica al otro como sujeto en la 
construcción de saber, desde los intereses de las comunidades y para la transformación de la realidad. Es un modo de investigar en el que el sentimiento, el pensamiento y la acción se conjugan para que la vida sea otra, se cambie, se haga para satisfacer las necesidades de las personas. El saber de los sectores populares es una forma de conocimiento que, desde lo inmediato, lo sensible, lo poético y lo imaginario hace que la práctica cotidiana, el quehacer concreto y la producción material se constituyan en elementos fundamentales para crear la vida colectiva. Es lo que Orlando Fals Borda llama ciencia popular: un conjunto de conocimientos y procedimientos para aprender del mundo y transformarlo desde la mirada de los sectores populares (Fals Borda, 1999). Teniendo en cuenta el carácter de I.A.P y de E.P de esta Propuesta Educativa, el desarrollo del trabajo de investigación se organizó en cinco fases que se entrelazan y se encuentran simultáneamente durante la investigación, la acción y la participación. Las fases se presentan desde la perspectiva académica y no como una organización cronológica y secuencial de los hechos, ya que en la vida cotidiana éstos se cruzan dependiendo del mismo desarrollo del proceso y como tal se ajustan a la dinámica propia de la investigación (Wittrock, 1989a; 1989b).

\subsection{Primera Fase}

Aproximación teórica a los temas de contexto político, económico y social especialmente en el terreno de la educación universitaria. La tarea inicial consistió en realizar lecturas teóricas que apoyaran la profundización y problematización del campo. Los criterios de selección de los documentos de archivo y actuales que se revisaron fueron: reconocimiento nacional e internacional de los autores como autoridades en la materia, la pertinencia y actualidad con relación a los temas. Esta revisión teórica se realizó desde un punto de vista crítico, interpretando las lecturas a la luz de su aporte a los intereses de esta investigación en seminarios profesorales semestrales y utilizando las más relevantes en la programación académica con los estudiantes que ingresaban a la línea cada año.

\subsection{Segunda Fase}

Realización de 12 entrevistas a profundidad para obtener los relatos de los actores clave: 7 a hombres y mujeres, representativas del sector popular-urbano-marginal (realizadas por una de las investigadoras principales como parte de su tesis doctoral, I-1), tres a educadores populares reconocidos por su trascendencia nacional e internacional (realizadas por uno de los investigadores como parte de su formación de maestría, I-2) y dos a los profesores 
fundadores y mayores impulsadores de la iniciativa (realizadas por el tercer investigador como proyecto de año sabático, 1-3). Esta fase consistió en invitar a los actores clave representativos para los fines de la investigación, contando con su libre participación y el deseo de aportar con sus relatos. Para este trabajo, las personas elegidas han mantenido por más de 5 y hasta 10 años, una estrecha relación afectiva y de constante participación con el equipo de investigación. La temática de la entrevista se anunció con suficiente antelación a través de una invitación verbal, en la cual se precisaban el día, la hora, los temas y el anuncio de algunos interrogantes que era necesario abordar durante la entrevista, así como el consentimiento informado. Las entrevistas fueron grabadas con el propósito de favorecer la recuperación y elaboración de las historias de vida en el marco de la investigación (Alonso, 1994; Cuevas, 2001; Molano, 1998).

\subsection{Tercera Fase}

Acompañamiento de I@s docentes a la realización de más de 30 trabajos de grado en modalidad e investigación cualitativa o mixta de los estudiantes participantes durante 12 años.

\subsection{Cuarta Fase}

Análisis e interpretación documental. Los documentos que enriquecieron el marco teórico, las entrevistas, los relatos de todos los participantes, los resultados de los trabajos de grado de los estudiantes y los diarios de campo de cada año académico, fueron analizados y leídos durante un año en la modalidad de Seminario Alemán por los tres investigadores principales, dos profesores y tres estudiantes participantes del proceso. Esta lectura se realizó críticamente a la luz del contexto nacional -que pasó de un conflicto armado de casi 60 años a un proceso de implementación del Acuerdo de Paz- y la recuperación de las historias de vida junto con la interacción de los participantes con los actores clave, todo lo cual permitió la estructuración de la propuesta educativa para la promoción de la vida y la paz. La tarea de analizar la información se realizó fundamentalmente sobre las categorías de análisis que fueron apareciendo en las sesiones de los seminarios. Las categorías de análisis más significativas se refieren a lo: político, popular, urbano, marginal, económico, cultural, al contexto social, la solidaridad, el trabajo, la protección, el afecto, el género, el entendimiento, la subsistencia, la libertad, las violencias, la identidad entre otras. El contenido analítico de la investigación dedicó especial atención a la lectura crítica del marco 
teórico y de las historias de vida surgidas de las narraciones de las entrevistas. De todas ellas emanan las ideas centrales que se tuvieron en cuenta para pensar los contenidos de la propuesta educativa.

\subsection{Quinta Fase}

Una vez realizado el análisis de los documentos, llevadas a cabo las entrevistas, recuperadas las historias de vida, hechas las lecturas críticas y recogiendo la experiencia de los años de docencia, investigación y extensión solidaria con los sectores populares, urbanos, marginales y comunidades indígenas de los tres investigadores principales, se presenta como conclusión y aporte de la investigación, la propuesta educativa con los sectores indígenas y populares-urbano-marginales que hemos denominado "Profesional de la Vida Comunitaria y la Paz" (Barahona, 2000; Mejía, 1999) y refleja la necesidad de una educación pública al servicio de las mayorías de los colombianos. La propuesta compromete a diferentes sedes, facultades y profesores de la Universidad, se han elaborado sus contenidos programáticos y se ha presentado en las diferentes instancias institucionales para su aprobación definitiva y posible puesta en marcha en 2021. Este proceso que ha durado casi tres años se encuentra en la etapa final que busca su aprobación e implementación por el Concejo Superior Universitario de la Universidad Nacional de Colombia, pretende desarrollarse en una de las sedes de frontera, precisamente denominada Sede La Paz, ubicada en una de las zonas más golpeada por el conflicto, donde la Universidad construyó y puso en marcha una de sus sedes hace dos años.

\section{CONCLUSIONES}

En la investigación se reconstruyeron historias de vida a través de narraciones que permitieron capturar la magia del relato, la fantasía del encuentro entre narrador y oyente. Las historias de vida de los participantes permitieron interpretar los significados de las situaciones particulares e individuales como reflejos y consecuencias de la historia del país en su variada geografía. Es decir, que el proceso y el resultado de las vidas de nuestros actores clave y de nosotros mismos, no es ajeno al contexto histórico, económico, político y social del país. Igualmente, ellos permitieron la comprensión de las realidades colombianas tales como la exclusión, el papel de los hombres y sobre todo de las mujeres en los sectores populares, las características del sector popular-urbano-marginal y de esta forma aportar al trabajo de extensión solidaria y educación popular de los docentes, desde la interacción social, hasta formas apropiadas de investigación transformadora para profesores y 
estudiantes interesados en comprender y aportar a la resolución de los problemas urgentes del país, en donde el más sentido es la violencia contra los líderes sociales y defensores de los derechos humanos, así como, la profunda injusticia social de carácter estructural en que viven los colombianos.

Con la investigación se hizo una aproximación global a la situación social y a la educativa superior colombianas para explorarlas, describirlas y comprenderlas de manera crítica y proponer a las autoridades universitarias un nuevo proyecto educativo a ser implementado en una de las que fueran zonas del conflicto. Lo anterior supone que las personas involucradas directamente en la investigación comparten un mismo contexto, no son sujetos aislados, sino que interactúan. Aunque cada contexto y experiencia social sea particular, guarda relaciones estructurales, históricas y culturales con conjuntos más vastos de población, de o que dieron cuenta los trabajos de grado realizados por los estudiantes, que clasificamos según su énfasis en: énfasis étnico, énfasis etnológico, énfasis en calidad de vida y vida buena, énfasis en educación popular, énfasis en economía popular, énfasis en desplazamiento forzado, énfasis en Derechos Humanos, Enfasis en bioética, énfasis en diseño curricular y énfasis en la reconstrucción del tejido social antes y después del Acuerdo de Paz entre la guerrilla de las F.A.R.C. y el Gobierno Colombiano.

La I.A.P y la E.P, como las hemos implementado y experimentado en este proyecto, pueden ser usadas en otros contextos para enriquecer y ampliar la perspectiva de la salud pública. La contextualizan según un lugar y un momento, un territorio y una época, la orientan según unos sujetos y unas circunstancias, una clase y una historia, la proyectan según unos intereses y unos deseos, una liberación y una humanización. La salud pública puede partir de la vida cotidiana y concreta de las mayorías excluidas y marginalizadas para crear condiciones de vida materiales dignas, humanas y justas.

Nos fuimos al maravilloso encuentro con las comunidades marginalizadas, con las mujeres del sector popular, con el inmenso mundo de saberes y formas de hacer la vida de las madres comunitarias, con la alegría de los niños y con las mil una anécdotas que vivíamos a diario en las calles, en los barrios, en las cocinas, en el fogón, en las asambleas, en la larga fila para recoger agua y en los generosos y pequeños espacios de las casas del sector. El trabajo de salud en paz nos invita a trabajar con las comunidades, con las organizaciones sociales, con los colectivos, con los educadores populares, en los lugares donde viven y abordando las necesidades que enfrentan. Es un ejercicio de construcción de soluciones en 
lo inmediato que buscan ir dando respuesta a las preguntas que la vida presenta. Es una creación conjunta, donde cada cual llega con lo que tiene y con lo que le falta y mancomunadamente se van creando los aprendizajes, las prácticas y las teorías sobre la vida, la paz y la salud. Nuestros estudiantes encontraban de todo en su trabajo comunitario acompañado por nosotros: economía informal, la que quisieran, trabajadores de todas las ramas de la economía, exceptuando la bancaria, ejemplos de fortaleza ante las tragedias humanas, capacidad de sus habitantes para superar el infortunio, la lluvia, el frío, el barro, los derrumbes, hermandad para ir construyendo las casas de familia en familia, pero ayudando todos, la minga. Sin idealismos, también sabíamos de las peleas, los golpes y los abusos. Es realmente sorprendente lo que ocurre en la cotidianidad de los sectores populares: la enorme capacidad de organización para legalizar los barrios, para mejorar sus viviendas y las condiciones de vida, la infatigable tarea diaria para resolver el hambre y la falta de abrigo, para apoyar a cada miembro de la familia. Las formas de resolver la escasez económica, las dificultades para ingresar y mantener escolarizados a los niños, los problemas propios de la adolescencia y la juventud en medio de tantas carencias.

El desempleo y las formas de acceder al trabajo asalariado, prácticamente imposible por fuera del servicio doméstico, de las celadurías o de la construcción (al destajo). En fin, lo enunciado es una pequeñísima muestra del enorme mundo en las localidades y municipios más pobres de Bogotá y del país; son innumerables las historias y relatos que las comunidades refieren al brindar apoyo a las familias desplazadas que todos los días llegan del campo, de la misma Bogotá o de poblaciones de todo el país, como resultado de la guerra, el paramilitarismo, el tráfico de droga y la expansión del latifundio. La universidad tiene el deber moral y ético de acercarse a las comunidades con el fin de aprender, saber, y conocer las realidades materiales, políticas, culturales y sociales, para poder contribuir a las soluciones: inmediatas, mediatas y futuras. Para esto existen recursos teóricos, prácticos y metodológicos. En América Latina nació uno de los grandes pensadores para el trabajo con los sectores más oprimidos, a través de la educación: Paulo Freire, educador de educadores, creador de sueños, quien con sus propuestas pedagógicas maravillosas da esperanza a los más explotados para beneficio de todos, Freire, con sus explicaciones y formas educativas aporta inmensamente al trabajo barrial, social, comunitario y universitario. Creó categorías de análisis y extraordinarias formas de entender la educación liberadora, de resistencia, de la esperanza y de la indignación, realmente un genio de la pedagogía y la conciencia de clase (Freire, 1975; Freire, 1997). 
Para fortuna de nosotros aprendimos de la mano y voz del maestro Orlando Fals Borda cómo investigar, cómo accionar y cómo participar sin invadir, con ética y enorme respeto para los pobladores de los sectores populares (Fals Borda, 1999). No como objetos de estudio sino como seres sentipensantes. Alfredo Molano fue quien directa y personalmente nos enseñó a hacer historias de vida y además compartió con nosotros sus formas de andar, de viajar y de investigar (Molano, 1998). Manfred Max Neef fue nuestro cómplice, lo admiramos y leímos muchas veces, su Economía Descalza y su Desarrollo a Escala Humana eran nuestros leitmotiv con cada nuevo grupo de estudiantes (Max Neef, 1987; Max Neef, 1989). Los problemas jurídicos nos los resolvía, ni más ni menos, que el gran Eduardo Umaña Luna, un colectivo de abogados y la O.N.G "Salud al Derecho". Fuimos unos afortunados, crecimos como intelectuales, como ciudadanos, con los infinitos saberes populares aprendimos a respetar a las comunidades desplazadas y excluidas; conocimos más nuestro país y aprendimos a amarlo y por esto mismo a defender a las personas de las comunidades pobres que luchan desde que nacen hasta que se mueren. Sin embargo ellas gozan con todo, hasta sacan risas de las desgracias.

Nuestro horizonte de sentido y comprensión de la investigación cualitativa y del deber ser de la universidad pública tomó otra dirección, realmente nos transformamos, entendimos que sin estudiar la historia, el contexto social, político y cultural que nos atraviesa y el modelo de producción capitalista no éramos capaces de comprender las realidades nuestras, como universitarios y las de ellos como comunidades despojadas de sus territorios, desplazadas y humilladas. El proceso de construcción de una propuesta de salud en paz parte de reconocer que las comunidades tienen un saber sobre sus condiciones de vida. Las comunidades poseen una lógica, una práctica y un trabajo de transformación de la realidad que es necesario que los centros de educación superior reconozcan y sobre el que pueden aprender para ampliar y orientar su compromiso social. No nos quedamos en los barrios marginales solamente, nuestras miradas se alejaron de Bogotá y por solicitud de los estudiantes indígenas iniciamos interacciones con las comunidades a las que ellos pertenecían: Zenúes en San Andrés de Sotavento; Wayúu en el Resguardo Rizzia Las delicias y Cabo de la Vela; indígenas Coys y Natagaimas descendientes de los pijaos en los Resguardos de Ortega, Coyaima y Natagaima; Indígenas Tycunas y Yucas del Amazonas en Puerto Nariño; Indígenas Xicuanis en el Meta; Indígenas Kankuamos y Aruhacos de la Sierra Nevada de Santa Marta, en el Caribe Colombiano. Otras narrativas, otros relatos y un mundo lleno de color, tradiciones, costumbres y formas de entender la paz, la salud, la 
enfermedad, las curas, los orígenes del planeta, etc. Aprendimos que las mujeres son en tanto están en una acción concreta en el mundo específico, en tanto actúan y transforman el mundo inmediato y material y no sólo si están en él o pertenecen a algo, por importante que parezca. Igualmente aprendimos que los indígenas son los mejores cuidadores de la Madre Tierra y nos dimos cuenta de que sus necesidades y carencias solo las puede resolver un modelo de sociedad profundamente humanista, respetuosa, y que el capitalismo es el enemigo de la vida planetaria por la voracidad del consumo, la centralización de la riqueza y la concentración de la producción, pasándole por encima a lo más sagrado: la vida digna, el medio ambiente sano y la conservación armoniosa entre hermanos humanos y la tierra poblada de verde y de diversidad. Aprendimos que la solidaridad es la ternura de los pueblos y no es un valor, ni un plusvalor como los muchos que pueblan la sociedad mercantil, sino que es una acción cotidiana en la cual sentimos y sabemos lo que es el hambre, la guerra y el miedo, y en consecuencia, hacemos todo lo posible para que nadie los sienta. Aprendimos que las mujeres han sido objetos de estudio y de teorías que hablan de ellas sin contar con sus voces. Aprendimos que la Universidad tiene una deuda gigantesca con el pueblo colombiano. No le podemos pedir que vuelva al sector popular o al indígena o a las veredas, porque, nunca ha estado, sólo ha pasado a tomar muestras para su conocimiento, olvidando que allí hay tantos saberes como en la selva que está pletórica de vida. Lo que sí le podemos pedir a la universidad es que se transforme en PLURIVERSIDAD, es decir, latinoamericana y popular. Porque nos guste o no, la mayoría de nuestro continente está conformado por hombres y mujeres del sector popular.

La salud pública tendría que acercarse decididamente a los sectores populares desde un horizonte de sentido político particular: la transformación de las condiciones de vida creadas por el modo de producción capitalista y reconocer el saber popular como una forma de construcción de mundo con la que es necesario ampliar su perspectiva de aproximación y de trabajo. Son estas opciones políticas y de saberes los aportes fundamentales de la E.P y la I.A.P al trabajo de la salud pública en nuestro continente. Como se constató en esta investigación, las condiciones de vida del pueblo colombiano son de extrema pobreza, surcadas por la guerra y la corrupción estatal, el fenómeno del desplazamiento forzado, el desempleo, las enormes inequidades en salud, educación y riquezas; ante este panorama, la educación pública no puede pasar despreocupada, debe sin demora asumir el papel que le toca jugar frente al pueblo colombiano, brindándole desde la docencia, la investigación y la extensión las herramientas éticas y políticas para lograr un mejor vivir. La Propuesta 
Educativa del Profesional de la Vida Comunitaria y la Paz ofrece las bases teóricas y metodológicas de carácter general e igualmente espera que los aportes de las otras disciplinas de nuestra Universidad, la enriquezcan y complementen.

Conocedores de la situación económica, política, social y cultural del país, la Universidad Nacional de Colombia, como la más importante institución educativa pública, debe formar sujetos solidarios antes que sujetos productivos y debe legitimar su conocimiento en la confrontación práctica a través del acompañamiento a las comunidades a quienes debe retribuir y acompañar para la superación de los problemas más sentidos y así colocarse a la altura académica que se merece. Para la realización de la Universidad que queremos en armonía con el país que soñamos es que se presenta este trabajo de investigación cualitativa, como una propuesta educativa fundamentalmente de extensión solidaria, que todas las carreras y disciplinas pueden retomar haciendo los ajustes necesarios en el terreno de la investigación y la formación. El Programa del Profesional de la Vida Comunitaria y la Paz, se basa en la reflexión, acompañamiento y participación de la Universidad en la construcción de alternativas de paz dentro de la creación de mejores condiciones de vida. Esto implica asesoría a las comunidades para la construcción participativa de planes de desarrollo comunitario, municipal, departamental y regional que concreten los anhelos de justicia, dignidad y solidaridad de los colombianos. Estas acciones responden al compromiso ético-político de la Universidad en relación con la transformación de las condiciones de injusticia, exclusión, empobrecimiento y pauperización que han determinado el desarrollo social, económico y político de los colombianos. Por lo tanto, los tiempos de la puesta en marcha del Programa se mueven en dos lógicas: la de la Universidad, marcada por sus ritmos académicos (docentes, investigativos y de extensión) y la de los procesos sociales a los cuales se vincula decididamente. La primera, se mide en semestres y años; la segunda, en lustros y décadas. El Programa, por lo tanto, ha de moverse en un constante ir y venir de estas lógicas, respondiendo a la temporalidad universitaria desde la temporalidad de los procesos sociales.

\section{REFERENCIAS}

Alonso, L. E. (1994). Sujeto y Discurso: el lugar de la entrevista abierta en las prácticas de la sociología cualitativa. In Métodos y Técnicas Cualitativas de Investigación en Ciencias Sociales. Juan Manuel Delgado (Coordinador). Madrid: Ed. Síntesis S.A.

Arnal, J, Del Rincón, D \& Latorre, A. (1992): Investigación educativa. Fundamentos y metodología. Barcelona: Labor.

Barahona, F. (2000): La Educación para la Paz: Un instrumento pedagógico para la transformación de la 
realidad, en Primer Congreso Nacional Universitario por la Paz. Tomo I. Bogotá: Universidad Nacional de Colombia.

Bautista, J. J. (2014). ¿Qué significa pensar desde América Latina?. Madrid: Ediciones Akal.

Bonilla, E. \& Rodríguez, P. (1995). La investigación en Ciencias Sociales. Más allá del dilema de los Métodos. Bogotá: Universidad de los Andes.

Bouché, H. (1994): “Etnografía, etnología y antropología (Un enfoque pedagógico)”. In López-Barajas Zayas, E. y Montoya Sáenz, J. (ed.): La investigación etnográfica. Fundamentos y técnicas. Madrid: UNED.

Carr, W.\& Kemmis, S. (1988). Teoría Crítica de la Enseñanza. Barcelona: Martínez Roca.

Cuevas, P. (2001). El relato y las historias de vida en el campo de las representaciones. In Aportes № 56 . Bogontá: Ed. Dimensión Educativa.

De Soussa Santos, B. \& Meneses, M. P. (2014). Epistemologías del sur (perspectivas). Madrid: Ediciones Akal.

Delgado, J. M. (Coord.) (1994):.Métodos y Técnicas Cualitativas de Investigación en Ciencias Sociales. Madrid: Síntesis.

Fals Borda, O. (1999). Orígenes universales y retos actuales de la I.A.P. Anal político, 38, 73-90.

Freire, P. (1975). Pedagogía del Oprimido. Bogotá: América Latina.

Freire, P. (1997): Pedagogía de la Esperanza. Bogotá: América Latina.

Goetz, J. P. \& Lecompte, M. D. (1988): Etnografía y diseño cualitativo en investigación educativa. Madrid: Ed. Morata.

Habermas, J. (1987). Teoría de la acción comunicativa. Tomos I y II. Madrid: Ed. Taurus.

Quevedo, E. (1992). El proceso salud-enfermedad: hacia una clínica y una epidemiología no positivistas. In Sociedad y Salud. Bogotá: Zeus editores.

Mariño, G. (1992). Aprender a Enseñar. Bogotá: Instituto Colombiano de Bienestar Familiar.

Mariño, G. (1994). El Diálogo Cultural: Reflexiones en torno a su fundamentación, su metodología y su didáctica. Material de apoyo, Maestría en Educación Comunitaria. Bogotá: Universidad Pedagógica Nacional.

Max Neef, M. (1986). Desarrollo a Escala Humana. Una opción para el futuro. Santiago de Chile: CEPAUR.

Max Neef, M. (1989). La Economía Descalza. Santiago de Chile: CEPAUR.

Mejía, M. R. el al. (1999): Educación para la paz: Una pedagogía para consolidar la democracia social y participativa. Colección Mesa Redonda. Bogotá: Cooperativa Magisterio.

Molano, A. (1998). Mi historia de vida con las historias de vida. In Los usos de la historia de vida en las ciencias sociales. Vol. 1. Madrid: Anthropos. pp. 102-111.

Taylor, S. J. \& Bogdan, R. (1986): Introducción a los métodos cualitativos de investigación. La búsqueda de significados. Buenos Aires: Paidós.

Wittrock, M. (ed.) (1989a): La Investigación de la enseñanza. I. Enfoques, Teorías y Métodos. Barcelona: Paidós/MEC.

Wittrock, M. (ed.) (1989b): La Investigación de la enseñanza. II. Métodos cualitativos y de observación. Barcelona: Paidós/MEC. 